\title{
EXTREME DOUBLY STOCHASTIC MEASURES WITH FULL SUPPORT
}

\author{
DAVID FELDMAN
}

(Communicated by R. Daniel Mauldin)

\begin{abstract}
We give an elementary construction of doubly stochastic measures on $I \times I$ with full support.
\end{abstract}

\section{INTRODUCTION}

Let $m$ denote Lebesgue measure on $I=[0,1]$, the unit interval. A probability measure $\mu$ on $I \times I$ is doubly stochastic if for any measurable $A \subseteq I$,

$$
\mu(A \times I)=\mu(I \times A)=m(A) .
$$

An $n \times n$ nonnegative matrix is doubly stochastic if its rows and columns all sum to 1 . A doubly stochastic measure (resp. $n \times n$ matrix) is extreme if it is not the positive linear combination of any pair of distinct doubly stochastic measures (resp. $n \times n$ matrices). An extreme measure (resp. $n \times n$ matrix) is just an extreme point in the appropriate convex set. It is well known that the extreme doubly stochastic matrices are exactly the permutation matrices, i.e., the matrices with exactly one nonzero entry in each row and column. In contrast to the sparseness of such matrices, extreme doubly stochastic measures may have full support. We construct such a measure. Other constructions are already known [L]; our goal is simplicity.

\section{Notation}

If $M=\left(a_{i j}\right)$ is a $2^{k} \times 2^{k}$ permutation matrix, let $\tau_{M}$ be the measure preserving transformation on $I$ defined by $M$. More specifically, when $m_{i j}=1$,

$$
\tau_{M}(x)=x+(j-i) \cdot 2^{-k} \text { for } i / 2^{k} \leq x<(i+1) / 2^{k} .
$$

Set $I_{m}^{k}=\left[m 2^{-k},(m+1) 2^{-k}\right) \subseteq I$, where $0 \leq m<2^{k}$. Set $B_{m, n}^{k}=I_{m}^{k} \times I_{n}^{k}$. We call these dyadic squares.

As usual, $\delta_{a, b}=1$ if $a=b$ and $\delta_{a, b}=0$ if $a \neq b$. 


\section{CONSTRUCTIONS WITH PERMUTATION MATRICES}

We describe a procedure to build new permutation matrices from old.

Given a $2^{k} \times 2^{k}$ permutation matrix $M$ and $h \geq k$, we construct a $2^{k+h} \times$ $2^{k+h}$ permutation matrix called $\gamma_{h}(M)=\left(a_{i, j}\right), 0 \leq i, j<2^{k+h}$.

First, let $M^{\prime}=\left(b_{i, j}\right), 0 \leq i, j, 2^{k+h}$ be the unique $2^{k+h} \times 2^{k+h}$ permutation matrix with $\tau_{M^{\prime}}=\tau_{M}$. (We obtain $M^{\prime}$ by replacing those entries of $M$ that are 1 by $2^{h} \times 2^{h}$ identity matrices and those entries that are 0 by $2^{h} \times 2^{h}$ zero matrices.)

Entries of $\gamma_{h}(M)$ of the form $a_{p \cdot 2^{k+h}+q, p^{\prime} \cdot 2^{k+h}+q^{\prime}}$ with $0 \leq p, q, p^{\prime}, q^{\prime}<2^{k}$ are defined equal to $\delta_{p, q^{\prime}} \delta_{q, p^{\prime}}$; for the remaining entries, set $a_{i, j}=b_{i, j}$.

Alternatively, we may describe $\tau_{\gamma_{h}(M)} . B=\bigcup_{s=0}^{2^{k}} I_{s 2^{h-k}}^{h}$ is an invariant set for $\tau_{M}$. Having fixed $h$, we will call $B$ the exceptional set for $\tau_{M}$. Note that its measure, $m(B)=2^{k-h}$, is small when $h$ is large. For $x \notin B$, we have $\tau_{\gamma_{h}(M)}(x)=\tau_{M}(x)$. On the other hand, for $0 \leq s, t<2^{k}, \tau_{\gamma_{h}(M)}$ brings $I_{s 2^{h}+t}^{k+h}$ to $I_{t 2^{h}+s}^{k+h}$ by translation. This makes it clear that $\gamma_{h}(M)$ is a permutation matrix. Moreover, $m\left(I_{s 2^{h-k}}^{h} \cap \tau_{\gamma_{h}(M)}\left(I_{t 2^{h-k}}^{h}\right)\right)=2^{-h-k}$, so certainly $m\left(I_{s}^{k} \cap \tau_{\gamma_{h}(M)}\left(I_{t}^{k}\right)\right) \geq$ $2^{-h-k}$.

\section{EXTREME DOUBLY STOCHASTIC MEASURES WITH FULL SUPPORT}

Let $M_{0}$ be any $2 \times 2$ permutation martix, and put $M_{i+1}=\gamma_{3 \cdot 4^{i}}\left(M_{i}\right)$. Then $M_{i}$ is a $2^{4^{i}} \times 2^{4^{i}}$ permutation matrix. Let $\tau_{1}, \tau_{2}, \ldots$ be the transformations defined by $M_{1}, M_{2}, \ldots$, and let $B_{1}, B_{2}, \ldots$ be the corresponding exceptional sets, as described above.

Then $m\left(B_{i}\right)=2^{-2 \cdot 4^{i}}$, hence $\sum_{i=1}^{\infty} m\left(B_{i}\right)<\infty$.

For $x \notin \bigcap_{i=1}^{\infty} \bigcup_{k=i}^{\infty} B_{k}$, the sequence $\left(\tau_{k}(x)\right)$ is finally constant, and if follows that $\tau(x):=\lim \tau_{k}(x)$ exists almost everywhere. Similarly, we have $m\left(\tau^{-1}(B)\right)=\lim m\left(\tau_{k}^{-1}(B)\right)=m(B)$ for any measurable subset $B$ of $I$.

Put $\tilde{B}_{i}=\bigcup_{k=i}^{\infty} B_{k}$. Then $m\left(\tilde{B}_{i}\right)<2 \cdot 2^{-2 \cdot 4^{i}} \leq 2^{-4^{i}}$.

For $0 \leq s, t<2^{4^{i}}$, we have $m\left(\tau_{i+1}^{-1}\left(I_{s}^{4^{i}}\right) \cap I_{t}^{4^{i}}\right) \geq 2^{-4 \cdot 4^{i}}=2^{-4^{i+1}}$. Since $\tau^{-1}\left(I_{s}^{4^{i}}\right) \cap I_{t}^{4^{i}} \supseteq \tau_{i+1}^{-1}\left(I_{s}^{4^{i}}\right) \cap I_{t}^{4^{i}} \backslash \tilde{B}_{i+1}$, it follows that $m\left(\tau^{-1}\left(I_{s}^{4^{i}}\right) \cap I_{t}^{4^{i}}\right)>0$, i.e., the image of Lebesgue measure on the graph of $\tau$, call it $\beta_{\tau}$, has full support.

It is well known that the image of Lebesgue measure on the graph of a measure-preserving transformation is an extreme doubly stochastic measure, so the example is complete.

In order that the exposition be entirely self-contained and because the method itself may have some interest, the remainder of this paper develops an alternative approach to verifying extremality. 


\section{A SUFFICIENT CONDITION FOR EXTREMALITY}

Let $\mu, \mu_{1}$, and $\mu_{2}$ be doubly stochastic measures where $\mu=\alpha \mu_{1}+(1-\alpha) \mu_{2}$, $\mu_{1} \neq \mu_{2}, 0<\alpha<1$. Interchange the roles of $\mu_{1}$ and $\mu_{2}$, if necessary, so that we may take $\alpha \in\left(0, \frac{1}{2}\right]$. Being distinct, $\mu_{1}$ and $\mu_{2}$ cannot agree on all dyadic squares, so

$$
\left|\mu_{1}\left(B_{m_{0}, n_{0}}^{k}\right)-\mu_{2}\left(B_{m_{0}, n_{0}}^{k}\right)\right|=\varepsilon>0
$$

for some $B_{m_{0}, n_{0}}^{k}$. Any doubly stochastic measure assigns $B_{m_{0}, n_{0}}^{k}$ a measure in the interval $\left[0,2^{-k}\right]$, since $B_{m_{0}, n_{0}}^{k}$ sits inside a horizontal (and a vertical) strip of measure $2^{-k}$. Then

$$
\mu\left(B_{m_{0}, n_{0}}^{k}\right) \in\left[\alpha \varepsilon, 2^{-k}-\alpha \varepsilon\right]
$$

To see this, consider the interval $J \subset\left[0,2^{-k}\right]$ whose endpoints are $\mu_{1}\left(B_{m_{0}, n_{0}}^{k}\right)$ and $\mu_{2}\left(B_{m_{0}, n_{0}}^{k}\right) . J$ has length $\varepsilon$. The distance from

$$
\mu\left(B_{m_{0}, n_{0}}^{k}\right)=\alpha \mu_{1}\left(B_{m_{0}, n_{0}}^{k}\right)+(1-\alpha) \mu_{2}\left(B_{m_{0}, n_{0}}^{k}\right)
$$

to the nearest endpoint of $J$ is precisely $\alpha \varepsilon$, since $\alpha \leq \frac{1}{2}$. So, a fortiori, it cannot be closer than $\alpha \varepsilon$ to the endpoints of $\left[0,2^{-k}\right]$.

Now there are four $(k+1)$-order dyadic squares contained in $B_{m_{0}, n_{0}}^{k}$. For at least one, call it $B_{m_{1}, n_{1}}^{k+1}$, we must have

$$
\left|\mu_{1}\left(B_{m_{1}, n_{1}}^{k+1}\right)-\mu_{2}\left(B_{m_{1}, n_{1}}^{k+1}\right)\right| \geq \varepsilon / 4
$$

by (1). Arguing as we did for (2) shows

$$
\mu\left(B_{m_{1}, n_{1}}^{k+1}\right) \in\left[\alpha \varepsilon / 4,2^{-(k+1)}-\alpha \varepsilon / 4\right]
$$

and continuing in this fashion yields a nested sequence

$$
B_{m_{0}, n_{0}}^{k} \supset B_{m_{1}, n_{1}}^{k+1} \supset \cdots \supset B_{m_{j}, n_{j}}^{k+j} \supset \cdots
$$

of dyadic squares such that

$$
\mu\left(B_{m_{j}, n_{j}}^{k+j}\right) \in\left[\alpha \varepsilon 4^{-j}, 2^{-(k+j)}-\alpha \varepsilon 4^{-j}\right] \text { for } j=0,1, \ldots
$$

We conclude that a sufficient condition for $\mu$ to be an extreme doubly stochastic measure is that there be no nested sequence as in (6) that satisfies (7) for any $\varepsilon, \alpha>0$. Strengthening (7) slightly produces a simpler sufficient condition that does not involve $k, \varepsilon$, and $\alpha$ and suffices for our purposes. We shall state it as a lemma. 
Lemma 1. Let $\mu$ be a doubly stochastic measure on $I \times I$, and fix constants $\kappa$, $\delta>0$. Assume that on any nested sequence of dyadic squares,

$$
B_{0,0}^{0} \supset B_{m_{1}, n_{1}}^{1} \supset \cdots \supset B_{m_{l}, n_{l}}^{l} \supset \cdots,
$$

the following condition fails for infinitely many $l$ :

$$
\mu\left(B_{m_{l}, n_{l}}^{l}\right) \in\left[\kappa 4^{-(1+\delta) l}, 2^{-l}-\kappa 4^{-(1+\delta) l}\right] .
$$

Then $\mu$ is extreme.

\section{APPLYING THE LEMMA}

Observe that if $M$ is a $2^{k} \times 2^{k}$ matrix and $g \leq k$, we have

$$
\beta_{M}\left(B_{s, t}^{g}\right)=m\left(I_{s}^{g} \cap \tau_{M}\left(I_{t}^{g}\right)\right) .
$$

From this we may deduce

$$
\left|\beta_{\gamma_{h}(M)}\left(B_{s, t}^{g}\right)-\beta_{M}\left(B_{s, t}^{g}\right)\right| \leq m\left(\left(I_{s}^{g} \cap \tau_{M}\left(I_{t}^{g}\right)\right) \Delta\left(I_{s}^{g} \cap \tau_{\gamma_{h}(M)}\left(I_{t}^{g}\right)\right)\right) \leq 2^{k-g-h},
$$

since $I_{t}$ is the union of $2^{k-g}$ intervals of length $2^{-k}$ and $\tau_{M}$ and $\tau_{\gamma_{h}(M)}$ agree on each interval outside a subinterval of length $2^{-k} 2^{h} / 2^{k}$.

In particular, we have

$$
\left|\beta_{M_{i+j}}\left(B_{s, t}^{4^{i}}\right)-\beta_{M_{i+j+1}}\left(B_{s, t}^{4^{i}}\right)\right| \leq 2^{-\left(2 \cdot 4^{j}+1\right) 4^{i}} .
$$

Using symmetric differences again shows that

$$
\left|\beta \tau\left(B_{s, t}^{4^{i}}\right)-\beta_{M_{i}}\left(B_{s, t}^{4^{i}}\right)\right| \leq \sum_{j=1}^{\infty} \leq 2^{-\left(2 \cdot 4^{j}+1\right) 4^{i}}<2 \cdot 2^{-3 \cdot 4^{i}} .
$$

Since $\beta_{M_{i}}\left(B_{s, t}^{4^{i}}\right)$ is either 0 or $4^{-i}$, we may apply the lemma.

\section{ACKNOWLEDGMENT}

We would like to thank Professor R. M. Shortt for suggestions related to an earlier version of this paper and the referee for many improvements. An earlier version of this paper was part of the author's dissertation at Wesleyan University under F. E. J. Linton.

\section{REFERENCES}

[L]

V. Losert, Counterexamples to some conjectures about doubly stochastic measures, Pacific J. Math 99 (1982), 387-397.

Department of Mathematics, University of New Hampshire, Durham, New Hampshire 